\title{
EMERGENCIA, ESTADO ACTUAL Y PERSPECTIVAS DEL ENFOQUE ONTOSEMIÓTICO EN EDUCACIÓN MATEMÁTICA
}

\author{
EMERGENCE, CURRENT STATUS AND PERSPECTIVES OF THE ONTO-SEMIOTIC \\ APPROACH IN MATHEMATICS EDUCATION \\ EMERGÊNCIA, ESTADO ATUAL E PERSPECTIVAS DA ABORDAGEM \\ ONTOSEMIÓTICA NA EDUCAÇÃO MATEMÁTICA
}

\author{
Juan D. Godino \\ Universidad de Granada, Granada, España \\ Recibido: 26/07/2021 - Aceptado: 08/09/2021 - Publicado: 24/01/2022 \\ Remita cualquier duda sobre esta obra a: Juan D. Godino. \\ Email:jgodino@ugr.es
}

\begin{abstract}
RESUMEN
El Enfoque Ontosemiótico de investigación en didáctica de la matemática (EOS) fue iniciado en la Universidad de Granada a principios de los noventa, como resultado de la interacción de investigadores de dicha universidad con los desarrollos teóricos de la didáctica de la matemática iniciados en Francia. Así mismo, la diversidad de teorías usadas para estudiar los procesos de enseñanza y aprendizaje de las matemáticas llevó a la convicción de la necesidad y utilidad de tratar de clarificarlas, compararlas y avanzar en su posible integración. La estrategia de articulación de las teorías y el desarrollo de la aproximación ontosemiótica al conocimiento matemático y su aprendizaje ha sido fruto del análisis racional de los fundamentos, cuestiones y métodos de diversos marcos teóricos existentes y de la aplicación de las herramientas teóricas que se fueron produciendo en diversos trabajos experimentales. En este artículo presento una síntesis histórica del origen y desarrollo del EOS, sus principales aportaciones para la investigación en didáctica de las matemáticas, su estado actual a nivel internacional y algunas reflexiones sobre nuevas cuestiones abiertas y las dificultades para abordarlas.
\end{abstract}

Palabras clave: Educación matemática; Enfoque ontosemiótico; Articulación de teorías; Síntesis histórica; Origen y desarrollo del EOS.

\begin{abstract}
The Onto-semiotic Approach to research in didactics of mathematics (OSA) was initiated at the University of Granada in the early nineties, as a result of the interaction of researchers at that university with theoretical developments in the didactics of mathematics initiated in France. Likewise, the diversity of theories used to study the processes of teaching and learning mathematics led to the conviction of the need and usefulness of trying to
\end{abstract}


clarify them, compare them and advance in their possible integration. The strategy of networking of theories and the development of the onto-semiotic approach to mathematical knowledge and learning has been the result of the rational analysis of the foundations, issues and methods of various existing theoretical frameworks and the application of the theoretical tools that were produced in diverse experimental researches. In this article, I present a historical synthesis of the origin and development of OSA, its main contributions to research in didactics of mathematics, the current state of the art at the international level and the current status of OSA.

Keywords: Mathematics education; Onto-semiotic approach; Networking theories; Historical synthesis; Origin and development of OSA.

\section{RESUMO}

A Abordagem Ontosemiótica da investigação em didática da matemática (EOS) foi iniciada na Universidade de Granada no início dos anos noventa, como resultado da interação dos investigadores dessa universidade com os desenvolvimentos teóricos na didática da matemática iniciados em França. Do mesmo modo, a diversidade de teorias utilizadas para estudar os processos de ensino e aprendizagem da matemática levou à convicção da necessidade e utilidade de tentar clarificá-los, compará-los e avançar na sua possível integração. A estratégia de articulação das teorias e o desenvolvimento da abordagem ontosemiótica do conhecimento e da aprendizagem matemática foi o resultado da análise racional dos fundamentos, questões e métodos dos vários quadros teóricos existentes e da aplicação dos instrumentos teóricos que foram produzidos no trabalho experimental. Este artigo apresenta uma síntese histórica da origem e desenvolvimento do EOS, as suas principais contribuiçóes para a investigação em didática da matemática, o estado atual da arte a nível internacional e o estado atual do EOS.

Palavras-chave: Educação matemática; Abordagem ontosemiótica; Articulação de teorias; Síntese histórica; Origem e desenvolvimento do EOS.

\section{INTRODUCCIÓN}

Desde el nacimiento de la Didáctica de la Matemática en los años 70, la comunidad de investigadores viene elaborando diversas teorías que permiten describir y explicar los fenómenos relativos a los procesos de enseñanza y aprendizaje de las matemáticas, y ayudan también a mejorar tales procesos (Grugeon-Allys et al., 2016). La complejidad de dichos fenómenos, los distintos factores que se deben tener en cuenta y la influencia de los diversos contextos culturales, desde los cuales se generan las teorías, explican su profusión, lo cual genera dificultades de comunicación y de capitalización de los conocimientos.

En este trabajo voy a presentar una síntesis de la emergencia, estado actual y perspectivas de futuro del Enfoque Ontosemiótico (EOS) del conocimiento y la instrucción matemáticos (Godino, 2002; Godino \& Batanero, 1994; Godino et al., 2007) elaborado como respuesta a la diversidad de teorías usadas en educación matemática. En Godino (2017a) se presenta el EOS como un sistema teórico modular, abierto e inclusivo que trata de proporcionar principios y herramientas metodológicas para abordar los problemas epistemológicos, ontológicos, cognitivos, instruccionales y ecológicos inherentes a los procesos de enseñanza y aprendizaje de las matemáticas. Desde su inicio, con el trabajo de Godino y Batanero (1994), el EOS viene construyendo un sistema teórico para la educación matemática que comparte en gran medida la posición señalada por Ruthven cuando sugiere, 
Esto implica adoptar un punto de vista modular, tanto con respecto a la descomposición de las teorias en componentes de herramientas analiticas y con respecto a la composición de herramientas provenientes de diferentes teorias; mediante la posibilidad de que una teoria tome prestadas herramientas de otra o de la improvisación de nuevos marcos que combinen herramientas de varias teorias fuente para abordar un nuevo tipo de cuestión o un tipo antiguo de cuestiones de una nueva manera. (Ruthven, 2014, p. 278)

La estrategia de clarificación, comparación, hibridación y construcción modular de teorías, desde una aproximación antropológica y semiótica, está en la base del EOS. Se asume la pertinencia y potencial utilidad de avanzar hacia la construcción de un sistema teórico, que permita abordar de manera articulada los problemas epistemológicos, ontológicos, semiótico-cognitivos y educativos implicados en la enseñanza y aprendizaje de las matemáticas. Se considera que la Didáctica es la disciplina tecnocientífica que asume la responsabilidad de dar una respuesta coherente a los problemas didácticos citados. Se asume, por tanto, una concepción ampliada de lo didáctico, como lo relativo a los procesos de enseñanza y aprendizaje, al saber y la práctica matemática (génesis, desarrollo, difusión, transposición y utilización), así como la optimización de dichos procesos en los contextos educativos.

El artículo incluye las siguientes secciones. En la sección 2 describo la emergencia histórica del EOS en el marco del programa de doctorado en didáctica de la matemática de la Universidad de Granada y de nuestro encuentro con la didáctica francesa en dicho contexto académico. En la sección 3 analizo el estado actual de desarrollo reflejado en las publicaciones incluidas en el repositorio web, http://enfoqueontosemiotico.ugr.es. En la sección 4 hago referencia a la proyección internacional del EOS, mencionando las publicaciones en inglés incluidas en el repositorio, la presencia en congresos internacionales, diversos metaanálisis de publicaciones y la creación de comunidades virtuales. El artículo finaliza con una síntesis e indicación de algunos retos pendientes.

\section{EMERGENCIA DEL EOS}

En el contexto académico del curso de posgrado de Teoría de la Educación Matemática, impartido en la Universidad de Granada desde 1988, tomé conciencia de la existencia de diversas teorías creadas para abordar los problemas didáctico-matemáticos, lo cual puede ser un factor positivo, dada la complejidad de tales problemas. No obstante, si cada teoría aborda un aspecto parcial de los mismos, con lenguajes y supuestos distintos, se pueden obtener resultados dispares o incluso contradictorios, que pueden dificultar el progreso de la disciplina. Me pareció necesario tratar de comparar, coordinar e integrar dichas teorías en un marco que incluyera de manera coherente las herramientas necesarias y suficientes para abordar el estudio de los problemas de investigación que plantea la educación matemática. Los avances que se pudieran lograr en la solución de estos problemas teóricos deberían tener 
efectos positivos en la educación matemática, principalmente a través de la mejora de los procesos de formación de profesores.

Un problema importante fue la clarificación de la noción de significado de un objeto matemático, su relación con otras nociones, como concepto y concepción, así como su relación con la noción de comprensión. La distinción entre los aspectos personales y los institucionales para la noción de significado se consideró esencial para articular las aproximaciones epistemológicas y cognitivas en educación matemática. Como un primer resultado de estas indagaciones publicamos el trabajo "Significado institucional y personal de los objetos matemáticos" (Godino \& Batanero, 1994), precedido por una presentación previa en el Colloque Vingt ans de didactique des mathématiques en France, celebrado en Paris en 1993 (Godino \& Batanero, 1993).

Inicialmente propusimos como noción básica para el análisis epistémico y cognitivo (dimensiones institucional y personal del conocimiento matemático) los sistemas de prácticas manifestadas por un sujeto (o compartidas en el seno de una institución) ante una clase de situacionesproblemas. Se asume de esta manera una visión antropológica (Wittgenstein, 1973) y pragmatista (Peirce, 1958) de las matemáticas; por tanto, la actividad de las personas para la resolución de problemas se considera el elemento central en la generación del conocimiento matemático, así como para su enseñanza y aprendizaje.

Sin embargo, en los procesos comunicativos que tienen lugar en la educación matemática, no solo hay que interpretar las entidades conceptuales, sino también las situaciones problemáticas y los propios medios expresivos y argumentativos, los cuales desencadenan procesos interpretativos. Se asume que la matemática no es solo una actividad humana sino también un sistema organizado de objetos culturalmente compartidos. Ello supone conocer los diversos objetos emergentes de los tipos de prácticas, así como su estructura. Llegamos a la conclusión de que es preciso estudiar con más amplitud y profundidad las relaciones dialécticas entre el pensamiento (las ideas matemáticas), el lenguaje matemático (sistemas de signos) y las situaciones problemas para cuya resolución se inventan tales recursos.

En consecuencia, en una segunda etapa (a partir de 1998) fue necesario diseñar modelos ontológicos y semióticos más detallados que el elaborado hasta dicha fecha, considerando que el problema epistémico-cognitivo no puede desligarse del ontológico. Por este motivo nos sentimos interesados en continuar con la elaboración de una ontología suficientemente rica para describir la actividad matemática y los procesos de comunicación de sus producciones. Esta ampliación del marco teórico se describe en el artículo "Un enfoque ontológico y semiótico de la cognición matemática" (Godino, 2002), donde se progresa en el desarrollo de una ontología y una semiótica específica para estudiar los procesos de interpretación de los sistemas de signos matemáticos puestos en juego en la interacción didáctica.

En la monografía “Teoría de las Funciones Semióticas. Un enfoque ontológico-semiótico de la cognición e instrucción matemática” (Godino, 2003) describo ampliamente el estado de desarrollo del 
EOS hasta dicha fecha, mostrando las fuentes documentales previas en las cuales se fundamentó, las herramientas elaboradas para el análisis del conocimiento matemático y sus implicaciones para el análisis de los procesos de instrucción matemática. La noción de función semiótica como entidad relacional básica entre objetos y significados se consideró esencial, razón por la cual se adoptó como descriptor clave de la teoría emergente. En trabajos posteriores se consideró que "Enfoque ontosemiótico" reflejaba con más amplitud el alcance del modelo de análisis del conocimiento matemático y los procesos de instrucción matemática, aunque este nombre no incorpora otros rasgos, como son los supuestos pragmatistas y antropológicos que también aportan rasgos característicos del EOS.

En la mencionada monografía describo con detalle la teoría de los significados de los objetos matemáticos, entendidos como sistemas de prácticas personales e institucionales, la tipología de objetos que intervienen en las prácticas matemáticas (presentados como componentes o elementos del significado del objeto), la relación del significado con las nociones de comprensión y competencia, las facetas duales desde las cuales se pueden contemplar las prácticas y los objetos, así como la noción de función semiótica y sus tipos. En el capítulo 8 de la monografía (Godino, 2003) describimos herramientas teóricas para el análisis de los procesos de instrucción matemática, con el objetivo puesto en el desarrollo de una teoría de la instrucción matemática significativa. Este componente educativoinstruccional del EOS fue posteriormente revisado, ampliado y publicado como artículo en Recherches en Didactiques des Mathématiques (Godino et al., 2006).

Como nuevas etapas en el desarrollo del EOS identificamos las siguientes:

- El análisis de la dimensión normativa (Godino et al., 2009) y metanormativa (D’Amore et al., 2007), donde identificamos las conexiones mutuas y complementariedades entre las nociones de contrato didáctico y norma sociomatemática. También proponemos un procedimiento sistemático para reconocer el sistema de normas y meta-normas que condicionan y hacen posible los procesos de instrucción matemáticos.

- La ampliación y sistematización de los criterios de idoneidad didáctica (Godino, 2013) introducida inicialmente en Godino (2003).

- La elaboración de un modelo de conocimientos y competencias del profesor de matemáticas basado en los supuestos y herramientas del EOS (Godino, 2009; Godino et al., 2017; PinoFan \& Godino, 2015).

\section{ESTADO ACTUAL}

En esta sección incluimos una breve descripción de los problemas, principios y herramientas teóricas elaboradas, siguiendo la síntesis incluida en Godino et al. (2020), así como las principales publicaciones donde tales nociones se han desarrollado y aplicado. Estas publicaciones están incluidas en las diversas entradas del repositorio web disponible en http://enfoqueontosemiotico.ugr.es (Figura 1). 


\section{Figura 1}

Repositorio web de publicaciones EOS (bttp:/lenfoqueontosemiotico.ugr.es)
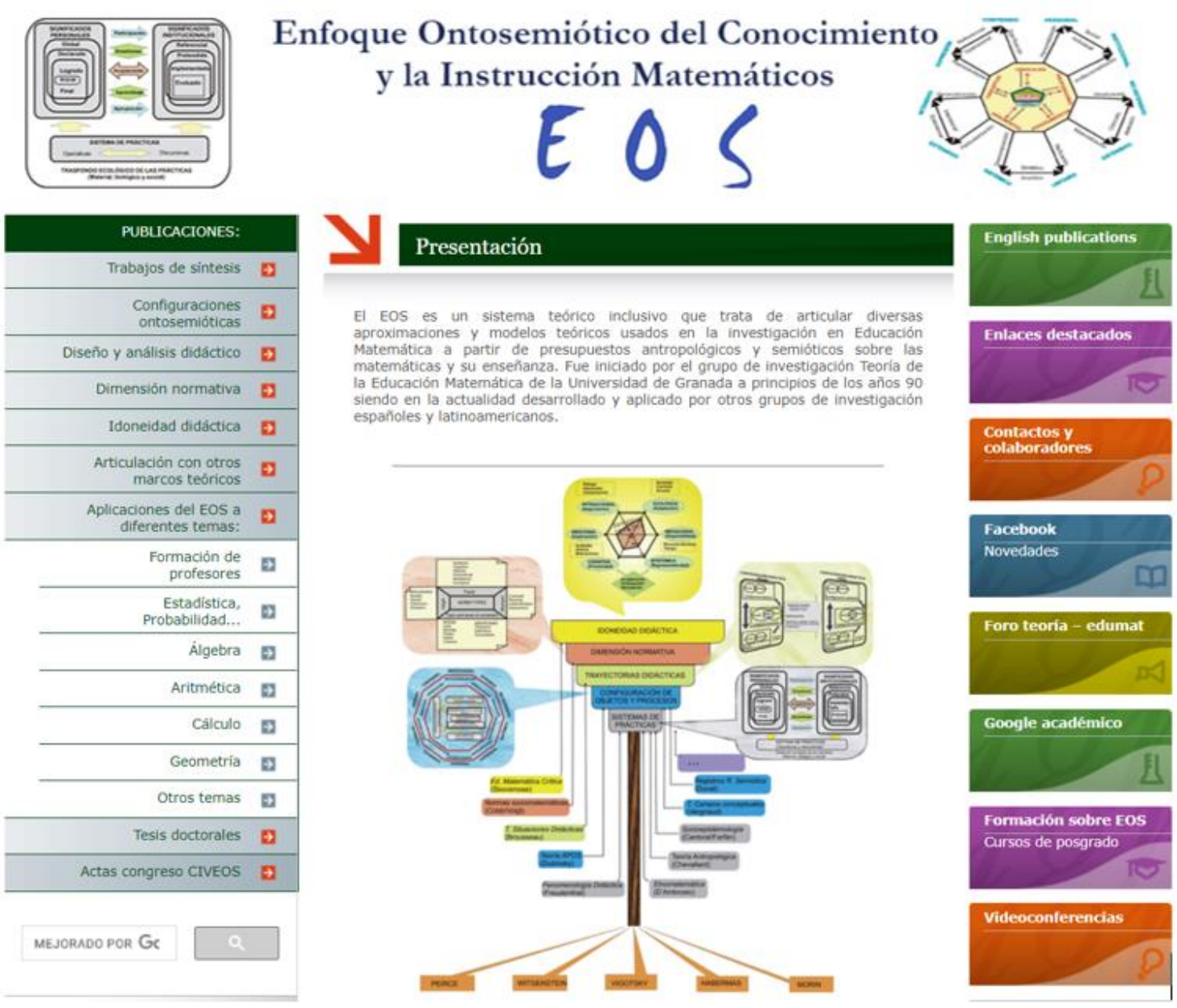

El EOS es un sistema teórico inclusivo que trata de articular diversas aproximaciones y modelos teóricos usados en la investigación en Educación Matemática a partir de presupuestos antropológicos y semióticos sobre las matematicas y su ensehanza. Fue iniciado por el grupo de investigación Teoria de espańoles y latinoamericanos. espanoles y latinoamericanos.
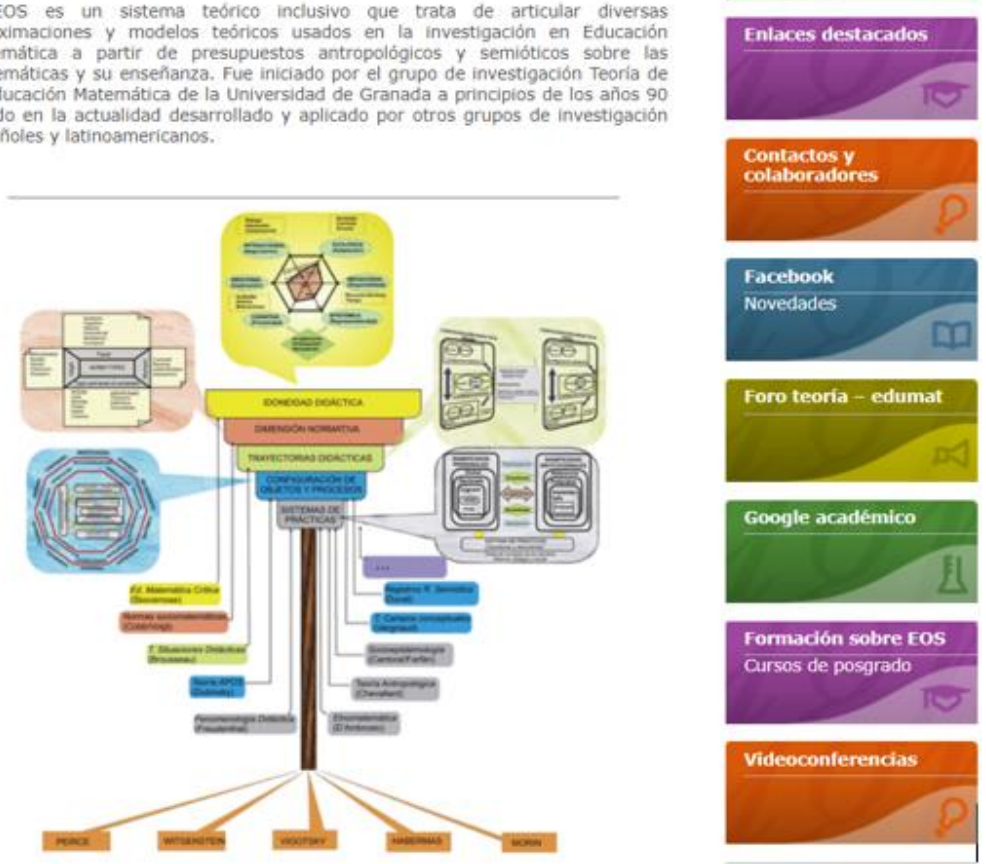

En la entrada Trabajos de sintesis del repositorio web (Figura 1) se incluyen varios artículos que presentan una perspectiva general de las nociones teóricas que componen el EOS, su motivación y supuestos antropológicos, pragmatistas y semióticos. Uno de ellos, el artículo Godino et al. (2007), publicado en la revista $Z D M$, ha sido uno de los más referenciados al presentar una síntesis global del EOS (1152 citas en Google Académico). La versión en portugués publicada en Acta Scientiae ha recibido 439 citas.

\section{PROBLEMAS EPISTEMOLÓGICOS, ONTOLÓGICOS Y SEMIÓTICO-COGNITIVOS DE LA EDUCACIÓN MATEMÁTICA}

Para abordar las cuestiones prácticas relacionadas con el diseño, implementación y evaluación de procesos de instrucción matemática de una manera fundamentada consideramos necesario plantear previamente problemas de índole teórica sobre la propia matemática, la naturaleza del conocimiento matemático y de su aprendizaje (Godino et al., 2020). Nos referimos al problema epistemológico, ¿Cómo emerge y se desarrolla la matemática?, al problema ontológico, ¿Quées un objeto matemático?, ¿Qué tipos 
de objetos intervienen en las prácticas matemáticas?, el problema semiótico-cognitivo, ¿Qué es conocer un objeto matemático?:Qué significa el objeto $O$ para un sujeto en un momento y circunstancias dadas?

Para dar respuesta al problema epistemológico se asume una visión antropológica (Wittgenstein, 1953) y pragmatista (Peirce, 1958) de las matemáticas; por tanto, la actividad de las personas en la resolución de problemas se considera el elemento central en la construcción del conocimiento matemático. Esta visión epistemológica se hace operativa en el EOS con la noción de práctica matemática y asumiendo su relatividad institucional y personal, lo cual lleva a asumir el siguiente principio epistemológico:

La matemática es una actividad humana centrada en la resolución de cierta clase de situaciones problemas. La realización de dicha actividad se concreta en la puesta en acción de sistemas de prácticas mediante las cuales se da respuesta a la situación-problema planteada.

Un segundo principio postula el carácter institucional y personal de las prácticas:

Las prácticas pueden ser idiosincrásicas de una persona o compartidas en el seno de una institución. No hay instituciones sin personas, ni personas desligadas de las diversas instituciones de las que de forma inevitable forma parte (familia, escuela, etc.).

Estos principios ligados a la cuestión epistemológica sobre la génesis del conocimiento dan lugar al siguiente método de indagación:

La génesis institucional del conocimiento matemático se investiga en el EOS mediante: 1) la identificación y categorización de las situaciones-problemas que requieren una respuesta; 2) la descripción de las secuencias de prácticas que se ponen en juego en la resolución.

La matemática, además de ser una actividad, es también un sistema lógicamente organizado de objetos; en consecuencia, es necesario plantearse el problema ontológico mencionado. Se asume que no hay actividad matemática sin objetos, ni objetos sin actividad. Como las prácticas pueden ser vistas desde la perspectiva social (prácticas institucionales, compartidas) o personal (prácticas individuales, idiosincrásicas), los objetos también pueden ser contemplados desde la dualidad institucional-personal, lo que origina el siguiente principio:

En las prácticas matemáticas intervienen diversas clases de objetos que cumplen diferentes roles: instrumental /representacional; regulativo (fijación de reglas sobre las prácticas), explicativo, justificativo. 
Dada la generalidad con la que se entienden las nociones de práctica y objeto, así como la gran diversidad de secuencias de prácticas (procesos) que se pueden realizar, se vio necesario y útil proponer una tipología de objetos y procesos básicos, que son los reflejados en la Figura 2, designada como configuración ontosemiótica. Estas configuraciones pueden ser epistémicas (redes de objetos institucionales) o cognitivas (redes de objetos personales). La herramienta configuración ontosemiótica incorpora de manera híbrida elementos de las nociones de concepto, concepción, esquema, praxeología matemática y registro de representación semiótica. En Godino et al. (2011) se presenta un desglose analítico de la noción de configuración ontosemiótica, tanto para los conocimientos institucionales como personales, con un ejemplo relativo al concepto de número natural. Así mismo, en Font et al. (2013) se analiza la emergencia de los objetos matemáticos a partir de las prácticas realizadas para resolver problemas matemáticos.

\section{Figura 2}

Configuración ontosemiótica de prácticas, objetos y procesos (Godino, 2014, p. 23)

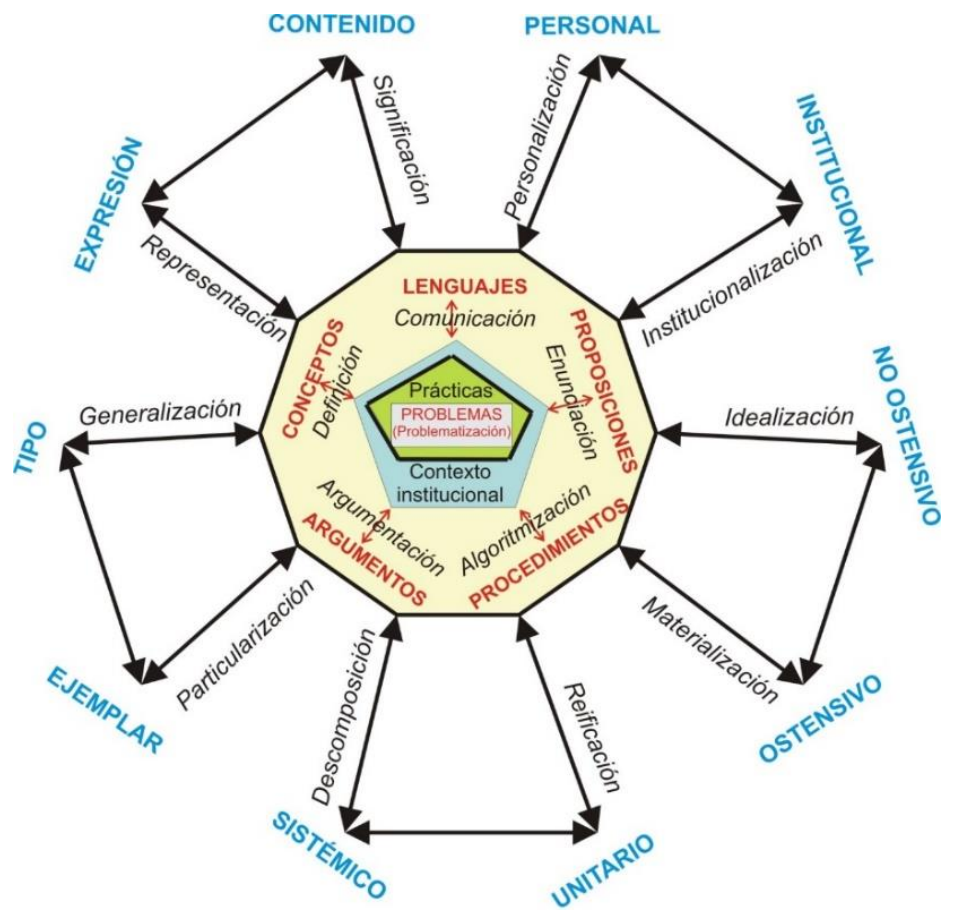

Para abordar el problema del significado de un objeto matemático para un sujeto en un momento y circunstancias dadas, esto es, el problema semiótico-cognitivo, se asume en el EOS que el conocimiento es el conjunto de relaciones que el sujeto (persona o institución) establece entre los objetos y las prácticas, relaciones que se modelizan mediante la noción de función semiótica. Hablar de conocimiento equivale a hablar del contenido de una (o muchas) función semiótica, resultando una variedad de tipos de conocimientos en correspondencia con la diversidad de funciones semióticas que se pueden establecer entre los diversos tipos de prácticas y objetos. De ahí, el siguiente principio: 
La correspondencia entre un objeto y el sistema de prácticas donde interviene tal objeto se interpreta como el "significado de dicho objeto" (institucional o personal).

Como base del análisis didáctico, se considera necesario reconstruir un significado global u holístico del objeto matemático mediante la exploración sistemática de los contextos de uso del objeto y los sistemas de prácticas que se ponen en juego para su solución. Dicho significado holístico se usa como modelo epistemológico y cognitivo de referencia de los significados parciales o sentidos que puede adoptar dicho objeto y constituye una herramienta metodológica para el análisis ontosemiótico de la cognición:

Un método para delimitar los diversos significados de los objetos matemáticos, y, por tanto, para la reconstrucción de los modelos de referencia epistemológica y cognitiva es el análisis de los sistemas de prácticas (personales e institucionales) y de las configuraciones ontosemióticas implicadas en los mismos.

En el repositorio del EOS se incluye la entrada "Significados y configuración ontosemiótica" con los artículos donde se desarrollan y ejemplifican las nociones de significado institucional y personal de los objetos matemáticos. En estos artículos se sientan las bases de una concepción antropológicapragmática y semiótica del conocimiento matemático, tanto desde el punto de vista institucional (social y cultural) como del personal (cognitivo). Así mismo, se desarrollan y ejemplifican las nociones de configuración de prácticas, objetos, procesos, así como la noción de función semiótica, que conjuntamente constituyen las herramientas analíticas de las prácticas matemáticas institucionales y personales. En esta entrada del repositorio se incluyen 26 artículos publicados en revistas indexadas (Recherches en Didactique des Mathématiques, Educational Studies in Mathematicas, Enseñanza de las Ciencias, International Journal of Science and Mathematics Education, International Journal of Mathematical Education in Science and Technology, REDIMAT, entre otras).

\section{PROBLEMA EDUCATIVO-INSTRUCCIONAL}

El modelo de instrucción que se asume en el EOS está basado en los principios de la psicología cultural/discursiva (Lerman, 2001; Radford, 2011), que atribuye un papel clave a la "zona de desarrollo potencial” (Vygotski, 1995). Contrariamente a los modelos constructivistas, la autonomía del estudiante en el proceso de aprendizaje es el resultado de dicho proceso y no un prerrequisito del mismo. No obstante, dado el papel central que la perspectiva antropológica del conocimiento da a los problemas y la actividad implicada en su resolución, la búsqueda, selección y adaptación de buenas situaciones problemas y la implicación de los estudiantes en su resolución es también un principio de la instrucción matemática significativa. Se deriva de este supuesto un modelo instruccional de tipo mixto (Figura 3), 
en el que la indagación y la transmisión del conocimiento se articulan de manera dialéctica (Godino \& Burgos, 2020); dicho modelo se resume en los siguientes principios:

- Se postula que el aprendizaje tiene como finalidad la apropiación por los estudiantes de los significados y objetos institucionales que le permitan afrontar la solución de determinados problemas y desarrollarse como persona.

- El estudio de los significados personales de los estudiantes es un componente esencial de la problemática educativa, ya que la apropiación de los significados institucionales pretendidos está condicionada por los significados personales iniciales de los estudiantes.

La noción de configuración didáctica constituye la principal herramienta metodológica para el análisis a nivel micro de los procesos de instrucción (Godino et al., 2007). Se define como cualquier segmento de actividad didáctica (enseñanza y aprendizaje) comprendido entre el inicio y fin del proceso de resolución de una situación-problema. Incluye, por tanto, las acciones de los estudiantes y del profesor, así como los medios planificados o usados para abordar la tarea. Otra herramienta metodológica para el análisis de la instrucción es la secuencia de configuraciones didácticas que constituye una trayectoria didáctica.

\section{Figura 3}

Modelo didáctico mixto indagativo-transmisivo (Godino E̊ Burgos, 2020, p. 97)

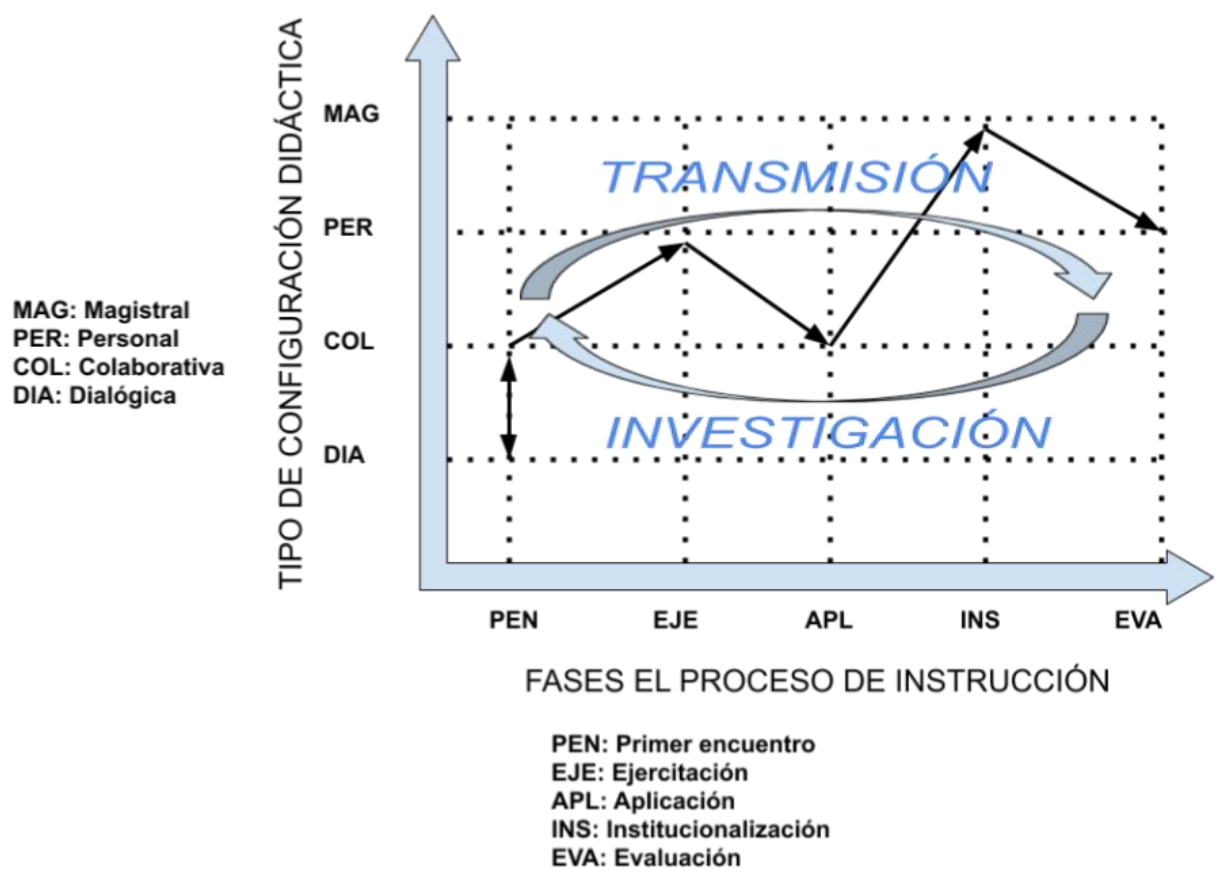


En el marco del EOS, el problema educativo-instruccional incluye al denominado problema ecológico, esto es, la indagación de los diversos factores y normas que condicionan los procesos de enseñanza y aprendizaje. En Godino et al. (2009) se aborda el estudio sistemático y global de las nociones de contrato didáctico y norma socio-matemáticas desde la perspectiva del EOS, tratando de identificar sus conexiones y complementariedades, y reconocer nuevos tipos de normas que faciliten el análisis de los procesos de enseñanza y aprendizaje de las matemáticas.

El problema educativo-instruccional también incluye el análisis y valoración de los procesos educativos desde el punto de vista de la optimización de los mismos, dado que el fin último de la investigación didáctica debe ser la mejora del aprendizaje, para lo cual es necesario contar con una serie de criterios de idoneidad que ayuden a lograr localmente dicha optimización.

Las publicaciones donde se aborda el problema educativo-instruccional en el EOS están agrupadas en tres entradas en el repositorio (Figura 1):

- Diseño y análisis didáctico: configuraciones y trayectorias didácticas; ingeniería didáctica basada en EOS. Se incluyen 20 artículos en revistas indexadas (Recherches en Didactiques des Mathematiques, Enseñanza de las Ciencias, AEIEM, Bolema, Infancia y Aprendizaje, Relime, entre otras);

- Dimensión normativa: sistema de normas que condicionan y posibilitan el desarrollo de los procesos instruccionales. Se incluyen tres artículos (Enseñanza de las Ciencias, Relime y Paradigma);

- Idoneidad didáctica: criterio sistémico de optimización de un proceso de instrucción matemática. Se incluyen 34 artículos en revistas indexadas (Bolema, Educaşão e Pesquisa, Acta Scientiae, Educación Matemática, Cultura y Educación, EURASIA Journal of Mathematics Science and Technology Education, AIEM, RELIME, entre otras).

\section{PROBLEMA DE FORMACIÓN DE PROFESORES}

En el marco del EOS se ha desarrollado un modelo teórico de conocimientos y competencias del profesor de matemáticas (modelo CCDM) (Godino et al., 2017) considerando que son claves para el profesor de matemáticas la competencia matemática y la competencia de análisis e intervención didáctica. El núcleo de la competencia de análisis e intervención didáctica consiste en diseñar, aplicar y valorar secuencias de aprendizaje propias, y de otros, mediante técnicas de análisis didáctico y criterios de idoneidad, para establecer ciclos de planificación, implementación, valoración y plantear propuestas de mejora. Para desarrollar esta competencia el profesor necesita, por una parte, conocimientos que le permitan describir y explicar lo que ha sucedido en el proceso de enseñanza y aprendizaje y, por otra, otros que le permitan valorar localmente lo que ha sucedido y hacer propuestas de mejora para futuras implementaciones. La competencia global de análisis e intervención didáctica del profesor de 
matemáticas está formada por cinco sub-competencias, las cuales están asociadas a cinco herramientas conceptuales y metodológicas del EOS: análisis de significados globales (basada en la identificación de situaciones-problemas y prácticas operativas, discursivas y normativas implicadas en su resolución); análisis ontosemiótico de las prácticas (identificación de la trama de objetos y procesos implicados en las prácticas); gestión de configuraciones y trayectorias didácticas (identificación de la secuencia de patrones de interacción entre profesor, estudiante, contenido y recursos); análisis normativo (reconocimiento de la trama de normas y metanormas que condicionan y soportan el proceso instruccional); y análisis de la idoneidad didáctica (valoración del proceso instruccional e identificación de potenciales mejoras).

En la entrada del repositorio web (Figura 1) hay incluidos 54 artículos en revistas indexadas sobre el tema de formación de profesores aplicando herramientas del EOS.

\section{PROBLEMA DE ARTICULACIÓN DE TEORÍAS}

La articulación de marcos teóricos (networking theories) está recibiendo una atención particular por diversos autores (Prediger et al., 2008; Radford, 2008), quienes consideran que la coexistencia de diversas teorías para explicar los fenómenos de una disciplina como la Didáctica de la Matemática, puede ser hasta cierto punto inevitable y enriquecedora, pero al mismo tiempo, puede constituir una rémora para su consolidación como campo científico. Ante la diversidad de teorías usadas en educación matemática nos parece necesario avanzar en la construcción de un sistema teórico que incluya las herramientas necesarias y suficientes, respetando el principio de parsimonia metodológica. Este problema se puede formular en los siguientes términos:

Dadas las teorias $T_{1}, T_{2}, \ldots . T_{n}$, focalizadas sobre una misma problemática de enseñanza $y$ aprendizaje de las matemáticas, ¿es posible elaborar una teoria T que incluya las herramientas necesarias y suficientes para realizar el trabajo de las $T_{i}(1 \leq i \leq n)$ ?

Este es otro de los problemas centrales que dio origen al EOS: el intento de comprender, comparar, coordinar e integrar teorías usadas en Didáctica de la Matemática, tales como, la Teoría de situaciones didácticas (TSD) (Brousseau, 2002), Teoría de los campos conceptuales (TCC) (Vergnaud, 1990), Teoría antropológica de lo didáctico (TAD) (Chevallard, 1992, 1999), Teoría de los registros de representación semiótica (TRRS) (Duval, 1996), entre otras.

En el repositorio del EOS (Figura 1) hay una entrada específica donde se incluye 14 artículos publicados sobre el problema de la articulación de EOS con otros marcos teóricos. 


\section{APLICACIONES DEL EOS A DIFERENTES CONTENIDOS MATEMÁTICOS}

En el repositorio web del EOS (Figura 1) se incluyen entradas a las publicaciones en revistas indexadas realizadas aplicando las distintas herramientas del EOS a los contenidos de Estadística, probabilidad y combinatoria, Álgebra, Aritmética, Cálculo, Geometría y Otros temas (Competencia y comprensión, Didáctica de las Ciencias Experimentales, Demostración y prueba, Educación infantil, Intuición en educación matemática, Lógica y algoritmia, Metacognición, Papel de las representaciones en educación matemática, Uso de metáforas en educación matemática).

\section{TESIS DOCTORALES Y ACTAS DE CONGRESOS}

El desarrollo y aplicación de las herramientas teóricas del EOS se ha realizado en el marco de diversos proyectos de investigación y programas de posgrado en diferentes universidades. En el repositorio web se han incluido hasta la fecha 90 tesis de doctorado. No se han incluido tesis de maestría, como tampoco se han incluido publicaciones en actas de congresos, exceptuando las Actas del Primer y Segundo Congreso Internacional Virtual sobre el Enfoque Ontosemiótico, celebrados en 2004 y 2017, respectivamente.

En la Tabla 1 incluimos el número de artículos incluidos en el sitio web del EOS según las diferentes herramientas usadas o el contenido abordado. También sugerimos un artículo en cada apartado como lecturas recomendadas para iniciar el estudio de cada apartado.

Tabla 1

Número de articulos y lectura inicial recomendada

\begin{tabular}{|c|c|c|}
\hline $\begin{array}{c}\text { Herramientas del EOS / } \\
\text { Aplicaciones }\end{array}$ & $\begin{array}{c}\text { № de } \\
\text { artículos }\end{array}$ & Lectura inicial recomendada \\
\hline Trabajos de síntesis & 15 & $\begin{array}{l}\text { Godino, J. D., Batanero, C., \& Font, V. (2020). El enfoque ontosemiótico: } \\
\text { implicaciones sobre el carácter prescriptivo de la didáctica. Revista Chilena de } \\
\text { Educación Matemática, 12(2), 3-15. }\end{array}$ \\
\hline $\begin{array}{l}\text { Significados y } \\
\text { configuraciones } \\
\text { ontosemióticas }\end{array}$ & 27 & $\begin{array}{l}\text { Godino, J. D., \& Batanero, C. (1994). Significado institucional y personal de } \\
\text { los objetos matemáticos. Recherches en Didactique des Mathématiques, 14(3), } \\
\text { 325-355. }\end{array}$ \\
\hline Diseño y análisis didáctico & 20 & $\begin{array}{l}\text { Godino, J. D., Contreras, A., \& Font, V. (2006). Análisis de procesos de } \\
\text { instrucción basado en el enfoque ontológico-semiótico de la cognición } \\
\text { matemática. Recherches en Didactiques des Mathematiques, 26(1), 39-88. }\end{array}$ \\
\hline Dimensión normativa & 3 & $\begin{array}{l}\text { Godino, J. D., Font, V., Wilhelmi, M. R., \& Castro, C. de (2009). Aproximación } \\
\text { a la dimensión normativa en Didáctica de la Matemática desde un enfoque } \\
\text { ontosemiótico. Enseñanza de las Ciencias, 27(1), 59-76. }\end{array}$ \\
\hline Idoneidad didáctica & 35 & $\begin{array}{l}\text { Godino, J. D. (2013). Indicadores de la idoneidad didáctica de procesos de } \\
\text { enseñanza y aprendizaje de las matemáticas. Cuadernos de Investigación y } \\
\text { Formación en Educación Matemática, 11, 111-132. }\end{array}$ \\
\hline $\begin{array}{l}\text { Articulación con otros } \\
\text { marcos teóricos }\end{array}$ & 15 & $\begin{array}{l}\text { Godino, J. D., Font, V., Contreras, A., \& Wilhelmi, M. R. (2006). Una visión de } \\
\text { la didáctica francesa desde el enfoque ontosemiótico de la cognición e } \\
\text { instrucción matemática. Revista Latinoamericana de Investigación en } \\
\text { Matemática Educativa, 9(1), 117-150. }\end{array}$ \\
\hline
\end{tabular}




\begin{tabular}{|c|c|c|}
\hline Formación de profesores & 55 & $\begin{array}{l}\text { Godino, J. D., Giacomone, B., Batanero, C., \& Font, V. (2017). Enfoque } \\
\text { ontosemiótico de los conocimientos y competencias del profesor de } \\
\text { matemáticas. Bolema, 31(57), 90-113. }\end{array}$ \\
\hline $\begin{array}{l}\text { Estadística, probabilidad y } \\
\text { combinatoria }\end{array}$ & $\begin{array}{c}35 \\
(\text { Estad.) } \\
18 \\
(\text { Prob.) } \\
3 \\
\text { (Comb.) }\end{array}$ & $\begin{array}{l}\text { - Arteaga, P., Batanero, C., Cañadas, G., \& Gea. M. (2012). Evaluación del } \\
\text { conocimiento especializado de la estadística en futuros profesores mediante } \\
\text { el análisis de un proyecto estadístico. Educação Matemática Pesquisa, } \\
\text { 14(2),279-297. } \\
\text { - Batanero, C. (2005). Significados de la probabilidad en la educación } \\
\text { secundaria. Revista Latinoamericana de Matemática Educativa, 8(3), } \\
\text { 247-263. }\end{array}$ \\
\hline Álgebra & 21 & $\begin{array}{l}\text { Godino, J. D., Aké, L., Gonzato, M., \& Wilhelmi, M. R. (2014). Niveles de } \\
\text { algebrización de la actividad matemática escolar. Implicaciones para la } \\
\text { formación de maestros. Enseñanza de las Ciencias, 32(1), 199-219. }\end{array}$ \\
\hline Aritmética & 5 & $\begin{array}{l}\text { Godino, J. D., Font, V., Wilhelmi, M. R., \& Arrieche, M. (2009). ¿Alguien sabe } \\
\text { qué es un número? UNIÓN, Revista Iberoamericana de Educación Matemática, } \\
\text { 19, 34-46. }\end{array}$ \\
\hline Cálculo & 17 & $\begin{array}{l}\text { Pino-Fan, L., Godino, J. D., \& Font, V. (2013). Diseño y aplicación de un } \\
\text { instrumento para explorar la faceta epistémica del conocimiento didáctico- } \\
\text { matemático de futuros profesores sobre la derivada (Parte 1). REVEMAT, } \\
8(2), 1-49 \text {. }\end{array}$ \\
\hline Geometría & 11 & $\begin{array}{l}\text { Godino, J. D., Cajaraville, J. A., Fernández, T., \& Gonzato, M. (2012). Una } \\
\text { aproximación ontosemiótica a la visualización en educación matemática. } \\
\text { Enseñanza de las Ciencias, } 30 \text { (2), 163-184. }\end{array}$ \\
\hline Otros temas & 26 & $\begin{array}{l}\text { Hummes, V. B., Font, V., \& Breda, A. (2019). Uso combinado del estudio de clases } \\
\text { y la idoneidad didáctica para el desarrollo de la reflexión sobre la propia práctica en } \\
\text { la formación de profesores de matemáticas. Acta Scientiae, 21(1), 64-82. } \\
\text { Font, V., Godino, J. D., \& D’Amore, B. (2007). An onto-semiotic approach to } \\
\text { representations in mathematics education. For the Learning of Mathematics, } \\
27(2), 1-7 \text {. } \\
\text { Font, V. (2007). Una perspectiva ontosemiótica sobre cuatro instrumentos de } \\
\text { conocimiento que comparten un aire de familia: particular-general, } \\
\text { representación, metáfora y contexto. Educación Matemática, 19(2), 95-128. }\end{array}$ \\
\hline
\end{tabular}

\section{PERSPECTIVA INTERNACIONAL DEL EOS}

\section{PUBLICACIONES EN INGLÉS}

En el repositorio web del EOS hay una entrada específica que incluye las publicaciones en inglés en las principales revistas de educación matemática, agrupadas en las mismas entradas que la página principal: trabajos de síntesis, significados y configuraciones ontosemióticas, diseño y análisis didáctico, idoneidad didáctica, articulación con otras teorías, formación de profesores, álgebra, aritmética, cálculo, estadística, probabilidad y combinatoria.

Una versión revisada del artículo seminal sobre EOS, Godino y Batanero (1994) fue publicada como capítulo del libro editado por Sierpinska y Kilpatrick (1998), con el título Clarifying the meaning of mathematical objects as a priority area of research in Mathematics Education. Una primera síntesis en inglés 
del EOS fue publicada en la revista ZDM - Mathematics Education (Godino et al., 2007); este artículo ha recibido 1201 citas en Google Académico y tiene un rango de citación en Springer Nature del 98\%.

\section{PRESENCIA EN CONGRESOS INTERNACIONALES}

La presentación de trabajos basados en EOS en los congresos latinoamericanos, Reunión Latinoamericana de Educación Matemática (RELME), la Conferencia Interamericana de Educación Matemática (CIAEM) y el Congreso Iberoamericano de Educación Matemática (CIBEM) ha sido analizada en Kaiber et al. (2017) para el periodo de 10 años anteriores a 2017. Se analizaron los últimos diez números disponibles de Acta Latinoamericana de Matemática Educativa-ALME, que contiene el conjunto de trabajos presentados en la RELME. En los últimos diez años se han realizado tres congresos del CIEAM, el XII en la ciudad de Querétaro, México, en 2007, el XIII en Belo Horizonte, Brasil, en 2011 y el XIV en Tuxtla Gutiérrez, México, en 2015. Sobre el CIBEM se tomaron para su análisis las actas de los tres últimos congresos, celebrados, respectivamente, en 2009 en Puerto Montt en Chile, 2013 en Montevideo en Uruguay y en 2017 en Madrid en España.

El análisis de las actas de estos congresos permitió identificar 188 artículos que toman el EOS como referencia teórica principal de la investigación o como guía teórica para la producción de análisis. Este conjunto de 188 publicaciones se compone de 121 artículos publicados en ALME, 26 en los Anales del CIAEM y 41 en los Anales del CIBEM, contemplando diferentes dimensiones o áreas de investigación de la Didáctica de las Matemáticas. Los trabajos publicados se refieren a las categorías Enseñanza y Aprendizaje y Formación de Profesores, estando más presentes las investigaciones en torno al proceso de enseñanza y aprendizaje de la Matemática de contenidos u objetos matemáticos específicos, los conflictos semióticos emergentes, la planificación y análisis de actividades, así como la discusión de criterios para la mejora de la enseñanza y el aprendizaje y la superación de dificultades. Los constructos del EOS aplicados fueron Configuraciones Ontosemióticas, Desarrollo y Análisis Didáctico e Idoneidad Didáctica, y los contenidos matemáticos refieren a Álgebra, Cálculo Diferencial e Integral, Estadística y Probabilidad, Geometría, Números y Operaciones y Resolución de Problemas. Los países representados son México (34,5\% de los casos), Venezuela (15,6\%), Colombia (14,4\%) Chile, Argentina, Brasil, Perú, Cuba, Guatemala, República Dominicana y Uruguay.

Godino y Contreras (2019) analizan el diseño e implementación de un dispositivo de estudio, basado en el uso de recursos virtuales, para presentar y discutir resultados de investigaciones sobre educación matemática basadas en la aplicación del EOS. Se trata del Segundo Congreso Internacional Virtual sobre el EOS (CIVEOS). Se describen los objetivos, antecedentes, los recursos humanos y técnicos empleados, así como los resultados obtenidos, en particular, se informa del número de inscritos (554), participantes activos (443), su distribución según países, estadísticas de las interacciones realizadas sobre las 120 contribuciones científicas presentadas y discutidas en los foros asincrónicos y mediante videoconferencias sincrónicas. Se finaliza con una síntesis de los resultados de la encuesta de evaluación 
respondida por una muestra de 99 participantes destacando las ventajas y debilidades del dispositivo virtual implementado, así como potenciales mejoras a introducir en futuras implementaciones. La celebración del congreso CIVEOS ha mostrado que la tecnología para la celebración de conferencias virtuales es relativamente simple y suficientemente madura para que pueda ser usada de manera intensa y efectiva en la difusión y construcción del conocimiento científico. Asimismo, ha permitido identificar con claridad el ámbito de difusión y relativa extensión de la comunidad de profesionales de la educación matemática interesados por el desarrollo y aplicación del EOS.

Adicionalmente, ha sido usual la presentación de trabajos apoyados en el EOS en los congresos CERME e ICME.

\section{META-ANÁLISIS DE PUBLICACIONES}

Breda et al. (2015) hacen una discusión teórica sobre la noción de idoneidad didáctica de los procesos de instrucción y cómo el uso de ésta se refleja en las investigaciones sobre la formación de profesores de Matemáticas. Se muestran los criterios de idoneidad y sus descriptores, abordándolos, primero, como un constructo teórico y, seguidamente analizan varias investigaciones que usan este constructo en el contexto de la formación de profesores de Matemáticas de diferentes niveles educativos y en países como España, Argentina, México, Chile y Brasil. La revisión bibliográfica realizada en este trabajo muestra que la noción de idoneidad didáctica está teniendo un impacto relevante en la formación del profesorado en diferentes países. Este impacto está relacionado con la idea de que uno de los componentes del conocimiento didáctico-matemático del profesor es el que permite evaluar y justificar la mejora de los procesos de enseñanza y aprendizaje de las matemáticas.

El uso de la noción de idoneidad didáctica ha sido también objeto de meta-análisis en el artículo de Malet et al. (2021). Se hace un análisis de contenido de 85 trabajos publicados entre 2005 y 2020, en español, portugués e inglés que usan esta herramienta teórica. Como resultado del análisis, se identifican nueve categorías emergentes que reflejan los distintos usos de la idoneidad en las investigaciones sobre educación. Las dos categorías que concentran más trabajos son la idoneidad como recurso para valorar procesos de instrucción y la que implica el desarrollo de la competencia de reflexión didáctica en la formación docente. Los resultados reflejan las tendencias en la agenda científica y eventuales líneas de investigación pendientes. Un punto particularmente interesante es que la idoneidad didáctica no se muestra como una herramienta cuyo uso solo está al alcance de los investigadores; las investigaciones muestran que también los profesores pueden usarla competentemente, sea para proponer mejoras justificadas a su práctica profesional, para seleccionar videos educativos o libros de texto con criterios científicos, o para valorar las producciones de sus alumnos, tareas específicas o incidentes didácticos puntuales.

El impacto del EOS en los posgrados de educación matemática desarrollados en Brasil ha sido objeto de análisis en el artículo de Breda et al. (2021). Realizan un meta-análisis de 16 tesis doctorales 
presentadas en el periodo 2005 a 2019 en distintas universidades brasileñas que utilizan las herramientas del EOS como marco teórico de referencia para el planteamiento del problema de investigación, así como el análisis e interpretación de los resultados. Observan que el período 2016 y 2017 fueron los años con mayor número de tesis depositadas; la mayoría de las tesis se realizaron en el estado de São Paulo y se enfocaron en cursos y formación docente. También observan que el tema más discutido fue la formación del profesorado y la herramienta más utilizada fueron los criterios de idoneidad didáctica, seguido de la configuración de objetos y procesos. Algunas tesis abordan también la articulación de EOS con otros enfoques teóricos.

\section{CREACIÓN DE COMUNIDADES}

Con el fin de complementar el repositorio web del EOS con un recurso interactivo se creó en 2015 una página Facebook ${ }^{1}$, seguida en la actualidad por 1335 personas.

Destacamos la creación en la Universidad de los Lagos (Chile) del Seminario Latinoamericano de Colaboración sobre el Enfoque Ontosemiótico (SLC-EOS) ${ }^{2}$. Este seminario es una iniciativa que surge a raíz del interés manifestado por los participantes del Grupo de Discusión de RELME «Incidencia en Latinoamérica de un Marco Teórico Inclusivo en la Investigación en Educación Matemática (GD-EOS)», que tuvo su primera reunión en 2016 en el marco de la RELME 30 (Monterrey, México) y continuó en los siguientes. El objetivo general del SLC-EOS es generar espacios para la colaboración, reflexión y discusión sobre el desarrollo y usos de las herramientas teórico-metodológicas del EOS en investigaciones, proyectos de posgrado, propuestas de enseñanza, tanto de los grupos de investigación como de profesores latinoamericanos, y para la formación de profesores.

\section{SÍNTESIS Y NUEVOS RETOS}

En el EOS se trata de avanzar en la construcción de un sistema de herramientas conceptuales y metodológicas que permitan hacer los análisis de nivel macro y micro de las dimensiones epistémica, cognitiva, instruccional y ecológica implicadas en los procesos de enseñanza y aprendizaje de las matemáticas, así como las interacciones entre las mismas. La noción general de objeto matemático, sus diferentes tipos, su relación con las prácticas matemáticas, las diferentes polaridades desde las que se pueden considerar y la noción de función semiótica configuran la Ontosemiótica, un modelo ontológico y semiótico que enriquece, complementa y articula las ontologías parciales que caracterizan otros modelos teóricos usados en educación matemática.

Se trata de un sistema teórico con carácter inclusivo, abierto y dinámico, fruto de la reflexión sobre distintos marcos teóricos usados en educación matemática, y apoyado en la realización de múltiples

\footnotetext{
${ }^{1}$ https://www.facebook.com/enfoqueontosemiotico2015

${ }^{2}$ http://edumat.ulagos.cl/seminarios/eos/
} 
investigaciones experimentales en el seno de diversos proyectos y programas de doctorado. Se tiene en cuenta las diferentes dimensiones y niveles de análisis requeridos por la investigación sobre los procesos de instrucción matemáticos en los diversos contextos. El EOS aporta herramientas para realizar un análisis didáctico integral que sirve de fundamento para el diseño, implementación y evaluación de los procesos instruccionales, teniendo en cuenta las diversas dimensiones, facetas, niveles y fases implicadas en dichos procesos.

Aunque se han publicado un número relativamente alto de trabajos con aplicaciones de la noción de idoneidad didáctica (Malet et al., 2021) reconocemos la necesidad de profundizar en su desarrollo, refinando los criterios e indicadores generales de idoneidad y desarrollando criterios específicos para diferentes contenidos. En cierta manera, los distintos marcos teóricos usados en educación matemática asumen de manera implícita criterios de idoneidad propios, como se analiza en Godino (2021) para el caso de la Teoría de situaciones didácticas, la Teoría antropológica de lo didáctico y la Educación Matemática Realista (van den Heuvel-Panhuizen \& Drijvers, 2014). No obstante, la clarificación y comparación de estos criterios de idoneidad de diferentes marcos teóricos y su articulación en un sistema coherente supone un programa de investigación que apenas sugiero en dicho artículo.

La articulación con otros marcos teóricos ha sido un tema central del EOS, como se refleja en las publicaciones realizadas; no obstante, es necesario continuar con esta problemática, analizando las concordancias y complementariedades como fuente de mutuo enriquecimiento. En este ámbito encontramos una dificultad crítica: la perspectiva holística del EOS, su carácter sistémico e inclusivo, basado en la asunción de presupuestos no solo ontosemióticos, sino también pragmatistas, antropológicos y socioculturales, plantea una dificultad en su relación con otros marcos teóricos. Parece natural, desde el punto de vista ecológico-social, que cualquier marco teórico, por ejemplo, la Etnomatemática o la Socioepistemología, defiendan su propia identidad ante los intentos de asimilación y acomodación por el $\mathrm{EOS}^{3}$.

Se han realizado algunas investigaciones en las que se aplican las herramientas del EOS al campo de la didáctica de la física. En el repositorio web hay incluidos tres artículos de revistas. Consideramos que un enfoque ontosemiótico, antropológico y pragmatista sobre el conocimiento y la instrucción, como propone el EOS, puede ser aplicado de una manera más amplia en el campo de la didáctica de las ciencias experimentales. Este es un campo de investigación en el que se puede profundizar en el futuro.

\section{ACLARATORIAS}

El autor no tiene conflictos de interés que declarar. El artículo ha sido realizado en el marco del proyecto de investigación PID2019-105601GB-I00, y con apoyo del Grupo de Investigación

\footnotetext{
${ }^{3}$ Véase el artículo “Articulación de teorías socio-cultuales en educación matemática desde la perspectiva ontosemiótica (Godino, 2017b).
} 
FQM-126 del PAI (Junta de Andalucía, España). El origen y desarrollo del EOS está estrechamente apoyado en el trabajo realizado por otros investigadores, en particular C. Batanero, V. Font, A. Contreras, M. R Wilhelmi, U. Malaspina, M. Pochulu, L. Pino-Fan, entre otros, a quienes expreso mi sincero reconocimiento.

\section{REFERENCIAS}

Breda, A., Font, V., \& Lima, V. M. R. (2015). A noção de idoneidade didática e seu uso na formação de professores de matemática. Jornal Internacional de Estudos em Educação Matemática, 8(2), 141. https://revista.pgsskroton.com/index.php/jieem/article/view/2364

Breda, A., Bolondi, G., \& de Abreu Silva, R. (2021). Enfoque ontossemiótico da cognição e instrução matemática: um estudo metanalítico das teses produzidas no Brasil. Revemop, 3, e202117. https://doi.org/10.33532/revemop.e202117

Brousseau, G. (2002). Theory of didactical situations in mathematics. Springer. https://link.springer.com/book/10.1007/0-306-47211-2

Chevallard, Y. (1992). Concepts fondamentaux de la didactique: perspectives apportées par une approche anthropologique. Recherches en Didactique des Mathématiques, 12(1), 73-112. https://revue-rdm.com/1992/concepts-fondamentaux-de-la-didactique/

Chevallard, Y. (1999). L'analyse des pratiques enseignantes en théorie anthropologique du didactique. Recherches en Didactique des Mathématiques, 19(2), 221-266.

https://revue-rdm.com/1999/1-analyse-des-pratiques/

D'Amore, B., Font, V., \& Godino, J. D. (2007). La dimensión metadidáctica en los procesos de enseñanza y aprendizaje de las matemáticas. Revista Paradigma, 28(2), 49-77. http://www.ugr.es/ jgodino/funciones-semioticas/dimension metadidactica 11nov07.pdf

Duval, R. (1996). Quel cognitif retenir en didactique des mathématiques?. Recherches en Didactique des Mathématiques, 16(3), 349-382. https://revue-rdm.com/1996/quel-cognitif-retenir-en/

Font, V., Godino, J. D., \& Gallardo, J. (2013). The emergence of objects from mathematical practices. Educational Studies in Mathematics, 82, 97-124. https://doi.org/10.1007/s10649-012-9411-0

Godino, J. D. (2002). Un enfoque ontológico y semiótico de la cognición matemática. Recherches en Didactiques des Mathematiques, 22(2-3), 237-284. https://revue-rdm.com/2002/un-enfoque-ontologico-y-semiotico/ 
Godino, J. D. (2003). Teoría de las funciones semióticas. Un enfoque ontológico semiótico de la cognición e instrucción matemática. Trabajo de investigación presentado para optar a la Cátedra de Universidad de Didáctica de la Matemática de la Universidad de Granada. http://www.ugr.es/ jgodino/funciones-semioticas/monografiatfs.pdf

Godino, J. D. (2009). Categorías de análisis de los conocimientos del profesor de matemáticas. UNIÓN: Revista Iberoamericana de Educación Matemática, 20, 13-31. https://www.ugr.es/ jgodino/eos/JDGodino\%20Union_020\%202009.pdf

Godino, J. D. (2013). Indicadores de la idoneidad didáctica de procesos de enseñanza y aprendizaje de las matemáticas. Cuadernos de Investigación y Formación en Educación Matemática, 11, 111-132. http://enfoqueontosemiotico.ugr.es/documentos/Godino 2013 idoneidad didactica.pdf

Godino, J. D. (2014, 24 de agosto). Sintesis del enfoque ontosemiótico del conocimiento y la instrucción matemáticos: motivación, supuestos y herramientas teóricas [presentación de diapositivas]. Repositorio web del EOS. http://enfoqueontosemiotico.ugr.es/documentos/sintesis EOS 2abril2016.pdf

Godino, J. D. (2017a). Construyendo un sistema modular e inclusivo de herramientas teóricas para la educación matemática. En J. M. Contreras, P. Arteaga, G. R. Cañadas, M.M. Gea, B. Giacomone, \& M. M. López-Martín (Eds.), Actas del Segundo Congreso International Virtual sobre el Enfoque Ontosemiótico del Conocimiento y la Instrucción Matemáticos (pp. 1-20). Departamento de Didáctica de la Matemática de la Universidad de Granada. http://enfoqueontosemiotico.ugr.es/civeos/godino.pdf

Godino, J. D. (2017b, 2 de agosto). Articulación de teorias socio-culturales en educación matemática desde la perspectiva del enfoque ontosemiótico [conferencia plenaria]. RELME 31: Reunión Latinoamericana de Matemática Educativa, Lima, Perú.

Godino, J. D. (2021). De la ingeniería a la idoneidad didáctica en educación matemática. Revemop, 3, e202129. https://doi.org/10.33532/revemop.e202129

Godino, J. D., \& Batanero, C. (1993). La notion de signifié en didactique des mathématiques. En M. Artigue, R. Gras, C. Laborde, \& P. Tavignot (Eds.), Colloque Vingt ans de didactique des mathématiques en France (pp. 218-224). La Pensée Sauvage éditions.

Godino, J. D., \& Batanero, C. (1994). Significado institucional y personal de los objetos matemáticos. Recherches en Didactique des Mathématiques, 14(3), 325-355.

https://revue-rdm.com/1994/significado-institucional-y/ 
Godino, J. D., \& Burgos, M. (2020). ¿Cómo enseñar las matemáticas y las ciencias experimentales? Resolviendo el dilema de la indagación y transmisión. Revista Paradigma, 41(Edición Cuadragésimo Aniversario: 1980-2020), 80-106. https://doi.org/10.37618/PARADIGMA.1011-2251.0.p80-106.id872

Godino, J. D., Batanero, C., \& Font, V. (2007). The onto-semiotic approach to research in mathematics education. ZDM: The International Journal on Mathematics Education, 39(1-2), 127-135. https://doi.org/10.1007/s11858-006-0004-1

Godino, J. D., Batanero, C., \& Font, V. (2020). El enfoque ontosemiótico: implicaciones sobre el carácter prescriptivo de la didáctica. Revista Chilena de Educación Matemática, 12(2), 3-15. https://doi.org/10.46219/rechiem.v12i2.25

Godino, J. D., \& Contreras, J. M. (2019). Uso de recursos virtuales en la difusión y construcción de conocimiento científico: el caso del congreso CIVEOS. Contextos de Educación, 26(19), 1-12. http://www2.hum.unrc.edu.ar/ojs/index.php/contextos/article/view/924/977

Godino, J. D., Contreras, A., \& Font, V. (2006). Análisis de procesos de instrucción basado en el enfoque ontológico-semiótico de la cognición matemática. Recherches en Didactiques des Mathematiques, 26(1), 39-88. https://revue-rdm.com/2006/analisis-de-procesos-de/

Godino, J. D., Font, V., Wilhelmi, M. R., \& Castro, C. de (2009). Aproximación a la dimensión normativa en didáctica de la matemática desde un enfoque ontosemiótico. Enseñanza de las Ciencias, 27(1), 59-76.

https://eprints.ucm.es/id/eprint/12634/1/Godino Font Wilhelmi DeCastro ES 2009.pdf

Godino, J. D., Font, V., Wilhelmi, M. R., \& Lurduy, O. (2011). Why is the learning of elementary arithmetic concepts difficult? Semiotic tools for understanding the nature of mathematical objects. Educational Studies in Mathematics, 77(2), 247-265.

https://doi.org/10.1007/s10649-010-9278-x

Godino, J. D., Giacomone, B., Batanero, C., \& Font, V. (2017). Enfoque ontosemiótico de los conocimientos y competencias del profesor de matemáticas. Bolema, 31(57), 90-113. https://doi.org/10.1590/1980-4415v31n57a05

Grugeon-Allys, B., Godino, J. D., \& Castela, C. (2016). Three perspectives on the issue of theoretical diversity. En A. Kuzniak, B. R. Hodgson, \& J-B. Lagrange (Eds.), The Didactics of Mathematics: Approaches and Issues (pp. 57- 86). Springer. https://doi.org/10.1007/978-3-319-26047-1 4 
Kaiber, C. T., Lemos, A., \& Pino-Fan, L. (2017). Enfoque ontossemiótico do conhecimento e da instrução matemática (EOS): um panorama das pesquisas na América Latina. Perspectivas da Educação Matemática, 10(23), 531-552.

https://periodicos.ufms.br/index.php/pedmat/article/view/5056

Lerman, S. (2001). Cultural, discursive psychology: a sociocultural approach to studying the teaching and learning of mathematics. Educational Studies in Mathematics, 47, 87-113.

https://doi.org/10.1023/A:1014031004832

Malet, O., Giacomone, B., \& Repetto, A. M. (2021). La idoneidad didáctica como herramienta metodológica: desarrollo y contextos de uso. Revemop, 3, e202110.

https://doi.org/10.33532/revemop.e202110

Peirce, C. S. (1958). Collected papers of Charles Sanders Peirce. 1931-1935. Harvard UP.

Pino-Fan, L., \& Godino, J. D. (2015). Perspectiva ampliada del conocimiento didáctico-matemático del profesor. Revista Paradigma, 36(1), 87-109.

http://revistaparadigma.online/ojs/index.php/paradigma/article/view/552

Prediger, S., Bikner-Ahsbahs, A., \& Arzarello, F. (2008). Networking strategies and methods for connecting theoretical approaches: first steps towards a conceptual framework. ZDM: The International Journal on Mathematics Education, 40(2), 165-178.

https://doi.org/10.1007/s11858-008-0086-Z

Radford, L. (2008). Connecting theories in mathematics education: challenges and possibilities. ZDM: The International Journal on Mathematics Education, 40(2), 317-327. https://doi.org/10.1007/s11858-008-0090-3

Radford, L. (2011). La evolución de paradigmas y perspectivas en la investigación. El caso de la didáctica de las matemáticas. En J. Vallès, D. Álvarez, \& R. Rickenmann (Eds.), L'activitat docent. Intervenció, innovació, investigació (pp. 33-49). Documenta Universitaria. http://www.luisradford.ca/pub/12 Girona Radford2011.pdf

Ruthven, K. (2014). From networked theories to modular tools? En A. BiknerAhsbahs, \& S. Prediger (Eds.), Networking of theories as a research practice in mathematics education (pp. 267-279). Springer. https://doi.org/10.1007/978-3-319-05389-9 16

Sierpinska, A., \& Kilpatrick, J. (Eds.) (1998). Mathematics education as a research domain: a search for identity. Kluwer, A. P. https://doi.org/10.1007/978-94-011-5194-8 
van den Heuvel-Panhuizen, M., \& Drijvers, P. (2014). Realistic mathematics education. En S. Lerman (Ed.), Encyclopedia of Mathematics Education (pp. 521-525). Springer.

https://doi.org/10.1007/978-94-007-4978-8 170

Vergnaud, G. (1990). La théorie des champs conceptuels. Recherches en Didactique des

Mathématiques, 10(2-3), 133-170.

https://revue-rdm.com/2005/la-theorie-des-champs-conceptuels/

Vygotski, L. (1995). Pensamiento y lenguaje (J. P. Tosaus Abadía, trad.). Paidós. (Original publicado en 1934).

Wittgenstein, L. (1973). Investigaciones filosóficas. Crítica.

\section{Cómo citar este artículo:}

Godino, J. D. (2022). Emergencia, estado actual y perspectivas del enfoque ontosemiótico en educación matemática. Revista Venezolana de Investigación en Educación Matemática (REVIEM), 2(2), e202201. https://doi.org/10.54541/reviem.v2i2.25 


\section{Juan D. Godino}

(c) (1)

Copyright $($ ) 2021. Juan D. Godino. Esta obra está protegida por una licencia Creative Commons 4.0. International (CC BY 4.0).

Usted es libre para Compartir — copiar y redistribuir el material en cualquier medio o formato - y Adaptar el documento - remezclar, transformar y crear a partir del material — para cualquier propósito, incluso para fines comerciales, siempre que cumpla la condición de:

Atribución: Usted debe dar crédito a la obra original de manera adecuada, proporcionar un enlace a la licencia, e indicar si se han realizado cambios. Puede hacerlo en cualquier forma razonable, pero no de forma tal que sugiera que tiene el apoyo del licenciante o lo recibe por el uso que hace de la obra.

$\underline{\text { Resumen de licencia - Texto completo de la licencia }}$ 